\title{
Causal Excitation in Antenna Simulations
}

\author{
Ioan E. LAGER \\ Faculty of EEMCS, Delft University of Technology, Mekelweg 4, 2628 CD Delft, the Netherlands \\ i.e.lager@tudelft.nl \\ Submitted January 29, 2021 / Accepted February 9, 2021
}

\begin{abstract}
The critical relevance of ensuring the excitation's causality in electromagnetic (EM) simulations is validated via theoretical arguments and simulation results. Two families of model pulses with an implicitly causal behavior, namely the windowed-power (WP) and the power-exponential $(P E)$ ones, are elaborately discussed. After introducing their unipolar prototypes, the relevant families are supplemented with monocycle and ringing variants, and are used for building signatures with almost rectangular spectral contents. Their utility is evidenced by contrasting their performance with that of other types of excitations that are habitually employed in antenna simulations. The WP pulse is also shown to be an almost exact replica of signatures generated by physical circuitry and to be singularly expedient for improving the effectiveness of EM computational packages.
\end{abstract}

\section{Keywords}

Antennas, causality, time-domain analysis, numerical analysis, pulse generation

\section{Introduction}

Causality is a fundamental property of macroscopic electromagnetic (EM) fields, stating that the electric field strength $\boldsymbol{E}(\boldsymbol{r}, t)$ and the magnetic flux density $\boldsymbol{B}(\boldsymbol{r}, t)$ at any instant $t$ are the effect of causes that acted before $t$ [1]. Causality, as accounted for throughout this study, clearly contrasts locality (or microcausality) that is a quantum theoretical concept. From a special relativity perspective, causality is a distinctive property of time-like phenomena (with classical EM falling decidedly under this category), as opposed to the space-like phenomena encountered within quantum mechanics [2]. From a theoretical viewpoint, causality is crucial to demonstrating the uniqueness of the EM initial value problem. In this respect, [3] has indisputably shown that uniqueness hinges upon the one-to-one correspondence between the causal time-domain (TD) EM field components and constitutive relations, on the one hand, and their time Laplace transforms, on the other hand (this argument will be reiterated later). Moreover, applying the reciprocity theorem (another fundamental EM result) to the case of un- bounded domains requires, again, the use of causal sources with a bounded spatial support [4].

The macroscopic EM field theory finds its prime practical application in the wireless (digital) transfer and, in particular, in antenna engineering (AE). The role played by causality in this case was stated in [5] that insisted on the detrimental effect of non-causal excitations in studies concerning antennas radiating in unbounded domains. Furthermore, [6] stressed the necessity to enforce causality for ensuring the physical realizability of any pulse.

Upon zooming-in on $\mathrm{AE}$, it is noted that the ultra wideband (UWB) technology is decisive to pursuing the advance in digital wireless applications [7], a trend that gained momentum after the Federal Communications Commission (FCC) released in 2002 the 3.1-10.6 GHz band for low-level, unlicensed use in UWB applications [8]. Due to the intrinsic technological intricacies in producing UWB (antenna) systems, their design and performance prediction depends critically on increasingly sophisticated software simulation tools. Many authors resort to this end to frequency-domain (FD) instruments - this choice is justified by compatibility with measurement equipment capabilities (network analyzers operate in FD) and by the use of traditional concepts in $\mathrm{AE}$, such as the operational bandwidth $B$. However, digital communication occurs essentially in TD, and overlooking this aspect may result into the well-known detrimental effect of intersymbol interference [9] that can render ineffective the communication in channels in which the radiated power levels are well above the expected signal-to-noise-and-interference (SNRI) thresholds. As a result, the EM simulation tools of choice within the realm of UWB antennas should be of the TD variety, with [10-12] offering relevant such examples. Surprisingly, these tools employed manifestly non-causal pulses as excitation: Gaussian pulses [10], or the square root raised cosine (SRRC) pulse [11,12]. In fact, although the intrinsic perils entailed by violating causality were already signaled in [13], non-causal excitations are still to this day the norm in TD EM simulations.

With this in mind, the present study will advocate the use of strictly causal excitations in TD EM simulations for (UWB) antenna design. This work relies on the author's quest for developing various classes of causal model pulses. Moreover, some of these pulses were cogently shown to (almost 
exactly) replicate pulses produced by (solid-state) pulse generators. For brevity, except for a small number of elements that were insufficiently covered in previous publications, the discussion will be confined to conceptual foundations, with technical details being intentionally left out - all these aspects are covered in great detail in the cited references.

After introducing some definitions, the account will proceed by introducing the two basic unipolar prototypes of the pulses to be discussed and their immediate monocycle descendants. Two uniquely opportune classes of pulses will be subsequently inferred from these prototypes. Some important implications of the use of these causal excitations will then be catalogued. Conclusions will be drawn at the end.

\section{General Definitions}

Throughout this study, position is specified by the coordinates $\{x, y, z\}$ with respect to a background Cartesian reference frame with origin $O$ and three mutually orthogonal unit vectors $\left\{\boldsymbol{i}_{\mathrm{x}}, \boldsymbol{i}_{\mathrm{y}}, \boldsymbol{i}_{\mathrm{z}}\right\}$ that, in this order, form a right-handed system. The position vector is $\boldsymbol{r}=x \boldsymbol{i}_{\mathrm{x}}+y \boldsymbol{i}_{\mathrm{y}}+z \boldsymbol{i}_{\mathrm{z}}$, with $|\boldsymbol{r}|=r$, and the time coordinate is $t$. (Partial) differentiation is denoted by $\partial$. The one-sided Laplace transform of a causal function $g(t)$ is

$$
\mathcal{L}[g](s)=\int_{t=0}^{\infty}[\exp (-s t) g(t)] \mathrm{dt},
$$

with $s \in \mathbb{R}$ and $s>0$. This choice ensures via Lerch's theorem [14], [15] that only one causal time-domain original corresponds to its related transform and is at the core of the uniqueness proof of the EM initial value problem [3]. The Fourier transform of a function $h(t)$ that satisfies the needed conditions is

$$
\mathcal{F}[h](\mathrm{j} \omega)=\int_{t=-\infty}^{\infty}[\exp (-\mathrm{j} \omega t) h(t)] \mathrm{dt},
$$

in which $\omega=2 \pi f$ (with $f \in \mathbb{R}$ being the frequency). Whenever applicable, $\mathcal{F}[h](\mathrm{j} \omega)$ will be inferred from (1) by taking $s=\mathrm{j} \omega$. For compactness, the alternative notation $\hat{H}$ will also be used for denoting the Fourier transform of $h(t) . H(\cdot)$ will denote the Heaviside unit step function.

\section{Causal Pulse Definitions}

As stated in the Introduction, at the core of this study are some pulse prototypes that, via suitable transformations, allow deriving classes of pulses of concrete practical relevance. The design of these model pulses relies on the general guidelines delineated in [16] namely:

- start from a strictly causal unipolar prototype;

- define that prototype by using an as small as possible number of parameters that should have a clear technical interpretation and, preferably, be easily put in correspondence with standardized definitions;
- ensure a controlled time-differentiability of the prototype - infinite time-differentiability is undesirable since it cannot be replicated via physical circuitry.

Starting from these precepts, this section will discuss two basic unipolar prototypes, namely the windowed-power (WP) and the power-exponential (PE) ones. Since antenna systems are practically always fed by means of signals with no DC component in their spectral diagram, immediate descendants of the prototypes will then be derived by time differentiation, this yielding the corresponding $\partial_{t} \mathrm{WP}$ and $\partial_{t} \mathrm{PE}$ monocycle signatures, respectively. This section is supplemented with a quick review of other causal pulses encountered in EM (numerical) analyses.

\subsection{Model Pulses with Finite Temporal Support}

Temporal boundedness is an important feature of pulsed feeding. For example, timed array antennas [17] use purposefully designed modulations of a single-tone feeding signal for shaping their radiation patterns. Inspired by a (mechanical) on/off switching, such arrays use standardly rectangular time-windowed sinusoidal excitations. However, one must observe that the far-field EM field radiated by antennas is at least the time-derivative of the feeding signal's signature [18] (the received signal in a loop-to-loop transfer being the third order time-derivative of the feeding current [19]). As a result, an on/off switched sine feeding renders the radiated EM field at least discontinuous, thus non-physical.

To remedy this situation, [21] introduced the windowedpower $(W P)$ unipolar prototype having the expression

$$
\mathrm{WP}(v, t)=t^{\prime v}\left(2-t^{\prime}\right)^{v} H\left(t^{\prime}\right) H\left(2-t^{\prime}\right)
$$

in which $v=2,3,4, \ldots$ is the pulse rising power and $t^{\prime}=t / t_{\mathrm{r}}$, with $t_{\mathrm{r}}>0$ being the pulse rise-time, namely the interval between the onset and the instant when the pulse peaks. This pulse is causal and has a finite temporal support $2 t_{\mathrm{r}}$. The support of its first $v$ time-derivatives is also $2 t_{\mathrm{r}}$. The pulse and its first $v-1$ time-derivatives are continuous at both onset and end, with the choice $v \geqslant 2$ ensuring this type of continuity at least for $\partial_{t} \mathrm{WP}(v, t)$. The WP prototype is normalized to unity. Another interesting feature of this model pulse is that its Fourier transform is analytical, namely

$$
\widehat{\mathrm{WP}}(v, \mathrm{j} \omega)=\exp \left(-\mathrm{j} \omega t_{\mathrm{r}}\right) t_{\mathrm{r}} 2^{v} v ! \sqrt{2 \pi} \frac{J_{v+1 / 2}\left(\omega t_{\mathrm{r}}\right)}{\left(\omega t_{\mathrm{r}}\right)^{v+1 / 2}}
$$

in which $J_{n+1 / 2}$ is the Bessel function of the first kind and fractional order [20, Section 10.1] (see the full proof of this result in Appendix 1). Note that $|\widehat{\mathrm{WP}}(\mathrm{j} \omega)|=|\widehat{\mathrm{WP}}(-\mathrm{j} \omega)|$ since $\operatorname{WP}(v, t)$ is real. Examples of both TD signatures and their corresponding spectra are given in [21]. Moreover, that publication also evidenced this pulse's exceptionally low spectral leakage $(\mathrm{SpL})$, an essential figure of merit of any apodization function [22].

A monocycle signature can be easily obtained from (3) by taking its time-differential. Its normalized expression is $\partial_{t} \mathrm{WP}(v, t)=N(v)\left(1-t^{\prime}\right) t^{\prime v-1}\left(2-t^{\prime}\right)^{v-1} H\left(t^{\prime}\right) H\left(2-t^{\prime}\right)(5)$ 
with $N(v)=2^{1-v}(v-1)^{1-v}(2 v-1)^{v-1 / 2}$. The WP monocycle has a zero-crossing at $t=t_{\mathrm{r}}$ and extrema at $t_{\mathrm{ex} ; \pm}=$ $t_{\mathrm{r}}\left[1 \pm(2 v-1)^{-1 / 2}\right]$, respectively. Its Fourier transform follows from (4), by multiplication by $N(v) \mathrm{j} \omega$. Examples for both TD signatures and their corresponding spectra can also be found in [21]. The $\partial_{t} \mathrm{WP}$ pulse was shown in [23] to almost perfectly replicate the signature generated by the solid-state pulse generator described in [24].

Higher-order time-differentiated versions of the WP pulse, as required, for instance, by evaluating the expressions in [19] or for implementing the algorithm in [25], can be easily derived. Here, the controlled differentiability of the prototype turns out to be extremely beneficial.

\subsection{Model Pulses with Infinite Temporal Sup- port}

New classes of causal pulses can be constructed by relaxing the temporal finiteness requirement. Two such classes were introduced in [16], out of which the power-exponential (PE) is highly relevant for AE applications. The unipolar prototype reads

$$
\mathrm{PE}(v, t)=t^{\prime v} \exp \left[-v\left(t^{\prime}-1\right)\right] H(t)
$$

in which $v$ and $t^{\prime}$ have the same significance as in (3). This pulse is also causal but has an infinite tail. Its conventional pulse width $t_{\mathrm{w}}$ defined according to [16, Eq. (23)] is interrelated with $v$ and $t_{\mathrm{r}}$ as

$$
t_{\mathrm{w}}=t_{\mathrm{r}} \frac{\Gamma(v+1) \exp (v)}{v^{v+1}}
$$

with $\Gamma(\cdot)$ denoting the Euler gamma function. This prototype too has controlled differentiability at its onset. Its Laplace transform is

$$
\mathcal{L}[\mathrm{PE}](s)=t_{\mathrm{r}} \frac{\Gamma(v+1) \exp (v)}{\left(s t_{\mathrm{r}}+v\right)^{v+1}} \text { for } \mathfrak{R}(s)>-v / t_{\mathrm{r}}
$$

with its Fourier transform $\widehat{\mathrm{PE}}$ following by taking $s=\mathrm{j} \omega$ in (8) - the condition $\mathfrak{R}(s)>-v / t_{\mathrm{r}}$ guarantees the validity of this choice. Examples of both TD signatures and their corresponding spectra are given in [16].

As with the WP, a monocycle is derived from (7) by taking the first time-differential, the relevant expression being

$$
\partial_{t} \operatorname{PE}(v, t)=N\left(1-t^{\prime}\right) t^{\prime v-1} \exp \left[-v\left(t^{\prime}-1\right)\right] H(t)
$$

in which the normalization by

$$
N(v)=v^{1 / 2}\left(\frac{v^{1 / 2}}{v^{1 / 2}-1}\right)^{v-1} \exp \left(-v^{1 / 2}\right)
$$

ensures a unit amplitude. The Laplace transform of $\partial_{t} \mathrm{PE}$ and, implicitly, its Fourier transform $\widehat{\partial_{t} \mathrm{PE}}$, follow by multiplying $\mathcal{L}[\mathrm{PE}](s)$ in $(8)$ by $N(v) s$. Examples of both TD signatures and their spectra are also given in [16].
Despite their infinite tail, the very simple expressions of these pulses made them attractive for several EM formulations. For example, the PE excitation was used in [26] and the $\partial_{t} \mathrm{PE}$ variant was used in $[25,27]$.

\subsection{Other Model Pulses Employed in Analytical EM Frameworks}

Apart from causality, the WP and PE pulses offer some additional beneficial features, such as the finite temporal support of WP, and their controlled differentiability at the onset (and the endpoint, for WP). However, their expression may be, occasionally, excessively complicated and other pulses may prove more appropriate.

A popular such choice is the triangular pulse, a superior alternative to the rectangular pulse with its jump discontinuities at endpoints. As with the WP and PE classes, the triangular pulse comes in unipolar and monocycle (bipolar) variants. While having an evidently simpler expression, the triangular signatures are discontinuous already in their first time-derivative, this making them unsuitable for formulations as that in [19]. However, its convolution with a large class of functions (for example testing functions in a Method of Moments context or a Green's function) can be carried out analytically, which is undoubtedly advantageous. As a result, the unipolar triangular excitation was used in $[28,29]$ while its bipolar variant was used in [30,31].

Another option is offered by the unipolar bell-shaped excitation. An appealing property of this type of excitation is that it can be obtained by convolving rectangular and triangular pulses [32,33] or by convolving two triangular pulses [34]. This procedure ensures a sufficient degree of smoothness at the pulse's endpoints. Another interesting observation is the remarkable similarity between the WP and $\partial_{t} \mathrm{WP}$ signatures, on the one hand, and (combinations of) suitably chosen unipolar bell-shaped excitations, on the other hand.

\section{Special Features of Pulsed Excita- tions}

In this section, two additional special features that can be provided by pulsed excitations will be examined.

\subsection{Pulses with Rectangular Spectral Content}

Electronic circuitry operates over a limited bandwidth, only. All pulses discussed in Sec. 3 have infinite spectra and, while expedient for numerical analyses, they cannot be exactly generated and manipulated by physical circuits. The intrinsic bandwidth limitation inherently affects wireless transmissions where it induces unwanted artifacts in the employed modulation schemes [9], with intersymbol interference as one of the most detrimental effects in digital communications. To control these artifacts, signals are filtered prior to modulation via filters with an as flat as possible transfer in the passband. 
Upon acknowledging the consequences of band limitation, antenna systems are often simulated via excitations having a spectral content that closely mimics a rectangular one. A customary choice in UWB antenna simulation is the square root raised cosine (SRRC) pulse [12] (that, in turn, is based on [9])

$$
\operatorname{SRRC}(t)=\frac{2 \beta}{\pi \sqrt{T_{\mathrm{S}}}} \frac{\cos \left[(1+\beta) \pi t^{\prime}\right]+\frac{\sin \left[(1-\beta) \pi t^{\prime}\right]}{4 \beta t^{\prime}}}{1-\left(4 \beta t^{\prime}\right)^{2}}
$$

where $T_{\mathrm{S}}=1 / R_{\mathrm{s}}$, with $R_{\mathrm{S}}$ being the symbol rate, $t^{\prime}=t / T_{\mathrm{s}}$, and $\beta$ is a dimensionless roll-off factor for bandwidth control. An example of the relevant TD signature and its spectrum is given in [12], respectively. The SRRC is not causal and it needs being truncated. This situation was highlighted in [9], that also commented that the truncation effect is acceptable within the realm of wireless communications due to the pulse's sharp decay. However, when used as an excitation in a TD EM simulation, turning on a SRRC via a Heaviside unit step function will result in a jump discontinuity (as it will be documented below) and this can severely impact on the validity of the obtained results.

Another quite widely employed band-limited pulse is the approximate prolate wave function [35] - this type of signature is more frequently used for windowing purposes. Nonetheless, this pulse is also non-causal and suffers from the same disadvantages as an SRRC excitation.

The need for causal pulses with an almost rectangular spectral content was resolved in $[5,21]$ that advocated a simple, but effective strategy for equipping the PE and WP families with this property. By denoting as $B$ an intended frequency bandwidth with upper and lower limits $f_{\mathrm{h}}$ and $f_{\mathrm{l}}$, respectively, and center frequency $f_{\mathrm{c}}=\left(f_{\mathrm{l}}+f_{\mathrm{h}}\right) / 2$, applying that strategy yielded the PE modulated-sinc-cosine (PE-sc) pulse [5]

$$
\begin{aligned}
& \mathrm{PE}-\mathrm{sc}\left(v, K_{\mathrm{sc}}, f_{\mathrm{c}}, t\right) \\
& =\operatorname{sinc}\left[K_{\mathrm{sc}}\left(t^{\prime}-1\right)\right] \cos \left[2 \pi f_{\mathrm{c}} t_{\mathrm{r}}\left(t^{\prime}-1\right)\right] \operatorname{PE}(v, t)
\end{aligned}
$$

and the WP modulated-sinc-cosine $(\mathrm{WP}-\mathrm{sc})$ pulse [21]

$$
\begin{aligned}
& \mathrm{WP}-\operatorname{sc}\left(v, K_{\mathrm{sc}}, f_{\mathrm{c}}, t\right) \\
& =\operatorname{sinc}\left[K_{\mathrm{sc}}\left(t^{\prime}-1\right)\right] \cos \left[2 \pi f_{\mathrm{c}} t_{\mathrm{r}}\left(t^{\prime}-1\right)\right] \mathrm{WP}(v, t) .
\end{aligned}
$$

$K_{\mathrm{sc}}$ in (12) and (13) is a scaling coefficient interrelating $B$ and $t_{\mathrm{r}}$ as $B=K_{\mathrm{sc}} / t_{\mathrm{r}}\left(K_{\mathrm{sc}} \geqslant 3\right.$ for practical applications). Examples of both TD signatures and their corresponding spectra are available in $[5,21]$. The therein provided examples concerned raising powers $v \geqslant 2$, such values being however recommendable for ensuring sufficient smoothness at onset (and the endpoint). The spectral behavior of both pulses was shown (i) to approximate increasingly well a rectangular shape as $K_{\mathrm{sc}}$ increases, while the influence of $v$ on its shape is minimal and (ii) to have an approximately $-6 \mathrm{~dB}$ attenuation at both $f_{\mathrm{l}}$ and $f_{\mathrm{h}}$. These properties immediately entailed easy design rules: by taking $K_{\mathrm{sc}} \geqslant 3, t_{\mathrm{r}}$ and $f_{\mathrm{c}}$ follow from the intended $f_{1}$ and $f_{\mathrm{h}}$, with $v$ being chosen more or less arbitrarily for ensuring a certain pulse 'smoothness'.

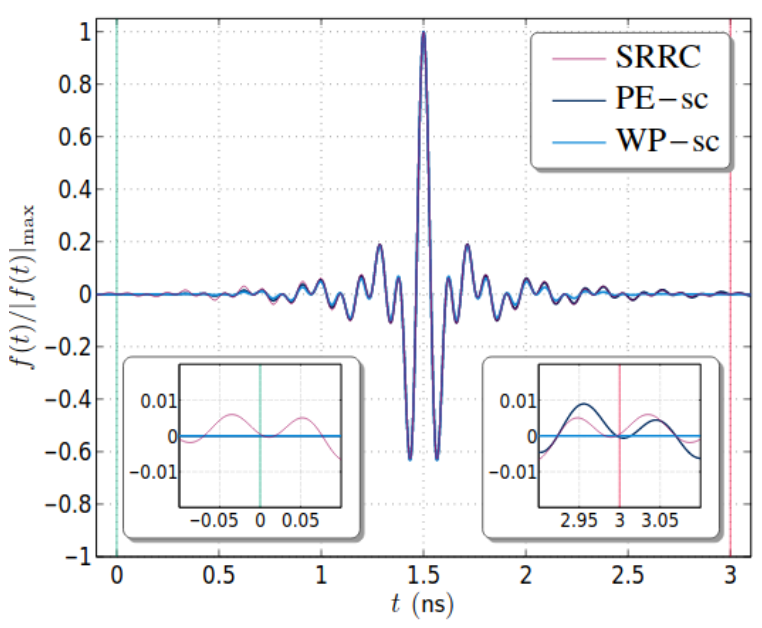

a

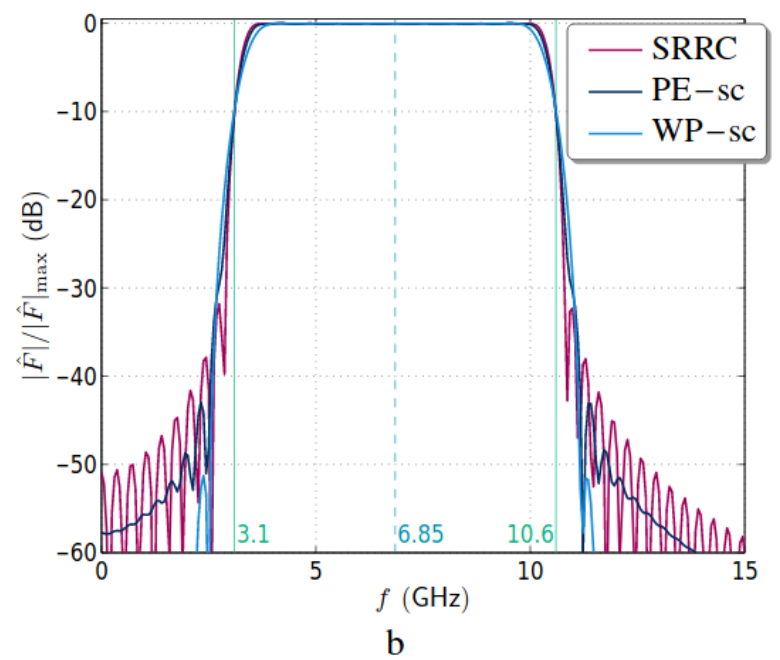

Fig. 1. Comparison between the SRRC pulse shown in [12, Figs. 1 and 2], and the PE-sc and WP-sc pulses mimicking its characteristics. (a) Time domain signatures; (b) spectral content. The solid vertical lines in (b) indicate the limits of the $-41.3 \mathrm{dBm}$ mask for indoor UWB systems, as specified in [36], and the dashed vertical line marks the targeted $6.85 \mathrm{GHz}$ center frequency. The insets in (a) are zoom-in around $t=0 \mathrm{~ns}$ and $t=2 t_{\mathrm{r}}=3 \mathrm{~ns}$.

The superiority of the WP-sc or PE-sc pulses over the SRRC one for (TD) EM numerical formulation ends is compellingly demonstrated in Fig. 1 that juxtaposes the excitation in [12] with $\mathrm{WP}-\mathrm{sc}$ and $\mathrm{PE}-\mathrm{sc}$ pulses tailored to provide an approximately flat spectral content over a frequency range between $f_{1}=3.1 \mathrm{GHz}$ and $f_{\mathrm{h}}=10.6 \mathrm{GHz}$ (corresponding to the $-41.3 \mathrm{dBm}$ part of the spectral mask for indoor UWB systems, as specified in [36]). Both the WP-sc and the PE-sc pulses have $t_{\mathrm{r}}=1.5 \mathrm{~ns}$ (tuned to the SRRC signature in [12]), $v=4$ and $K_{\mathrm{sc}}=10.9$, the last parameter entailing a nearperfect match of the $-6 \mathrm{~dB}$ points in the spectral diagrams of all 3 pulses. The plots demonstrate the remarkable concurrence between the in-band spectral contents of all three pulses. Nonetheless, the spectral leakage is largely improved, from $-44 \mathrm{~dB}$ for SRRC, to $-52.2 \mathrm{~dB}$ for PE- sc and to an exceptionally low $-64.7 \mathrm{~dB}$ for $\mathrm{WP}-\mathrm{sc}$. The benefits are most apparent in the TD signatures in Fig. 1(a). While the overall 
behavior is largely similar, the non-causality of the SRRC is evident in the zoom-in around $t=0$. Although one may argue that the SRRC value at 0 is small, its patent deviation in even a minute vicinity of 0 implies that any inaccuracy in its turning on may lead to significant step discontinuities. Here, one must recall that marching-on-time numerical schemes will demand staggered time sampling and, thus, some numerically evaluated values are bound to be affected by the relevant step discontinuities. A similar situation is apparent at $t=t_{\mathrm{r}}$ that, apart from illustrating the inferiority of a SRRC excitation, also highlights the benefit of the time-windowed $\mathrm{WP}-\mathrm{sc}$ pulse.

\subsection{Ringing Pulses}

Ringing is frequently manifest in feeding circuits and antennas - such occurrences are common in pulsed radar applications but are also likely to surface in baseband, digital transfer (see [37]). For facilitating the study of this phenomenon in antenna (numerical) experiments, [16] endowed the PE family with a ringing pulse defined as an amplitudemodulated cosine or sine function of carrier frequency $f_{0}$, its envelope being provided by the PE unipolar signature in (6). For increased flexibility, a normalized time-derivative variant of this pulse was also introduced. Examples of both TD signatures and their spectra are available in [16].

Although the mathematical expression of these pulses is rather intricate and, thus, their use in analytical formulations may be cumbersome, they prove to be extremely convenient for purely numerical formulations. Moreover, these pulses were shown in [16] to present a high degree of similarity with pulses effectively generated by physical circuits.

A ringing pulse based on the WP unipolar prototype was not yet presented in the literature. For maintaining this account's focus, this topic is deferred to future publications.

\section{Feature Practical Benefits}

Sections 3 and 4 focused on the conceptual benefits of using the causal WP and PE families of pulses and highlighted their propitious effect when used in certain computational EM frameworks. This section will concentrate on practical situations that can particularly leverage the characteristic properties of these families.

\subsection{Time-Windowed EM Simulations}

Present-day antenna designs critically depend on numerical studies of increasing complexity, with commercial EM computational tools becoming an omnipresent element of any design methodology. Such approaches push the available hardware resources to their limits and drastic simplifications must often be accepted for making those simulations tractable. One of the additional, significant complications induced by antenna simulations is the necessity to ensure their unimpeded radiation into an unbounded embedding. This is particularly testing in the case of the finite-difference or finite-element (type) methods that can only be applied to bounded domains of computations. Several strategies were proposed for precluding reflections from the boundary, the most popular being the absorbing boundary conditions introduced in [38] (and complemented in [39]) and the perfectlymatched layers (PML) introduced in [40-42] (with its coordinate stretching alternative [43]). From the two, the later has become the norm in most of the prevalent (commercial) software packages. However, as shown in [41], these domain termination methods are plagued by spurious reflections, primarily from waves reaching the boundary at slanting angles. Moreover, the specific transformations required by implementing these methods are only compatible with one single type of embedding, this all but ruling out the direct incorporation of multi-layer configurations that can only be reliably examined by introducing a homogeneous buffer between a truncated version of the layered arrangement and the PML boundary. Apart from the evident perturbation of the investigated structure, this choice places an additional penalty on the computational resources that need to also calculate field values in the essentially useless buffer zone.

An elegant remedy to this deficiency was advocated in [21] that put forward the use of time-windowed EM simulations. That approach relies on performing the analysis within a time-window that is sufficient for allowing the EM perturbation to propagate through the region of interest $\mathcal{D}$, but ends prior to any reflection propagating back from the boundary reached $\mathcal{D}$. Admitedly, this strategy does require extending the domain of computation for ensuring the needed safe margin, but (i) allows using elementary boundary conditions (PML-type boundaries are superfluous) and (ii) makes use of a limited time window, whereas traditional TD analyses require long simulations for ensuring that the entire input energy effectively leaves the domain of computation. Moreover, the approach is well-suited for examining layered configurations, providing the diameter of $\mathcal{D}$ is reasonably small. The essential ingredient for its application is the use of the causal, time-limited WP excitation.

Time-windowed EM simulations were shown in [21] to provide excellent results, including for frequency-domain (FD) studies, while requiring up to 30 times shorter computation times and comparable memory resources. This approach was also used for the numerical modeling of CMOS embedded radiating loops of the kind appearing in [25] - a typical multy-layered configuration example.

\subsection{Replication of Effectively Generated Pulses}

Both the WP and the PE families of causal pulses were introduced purely mathematically. While their suitability for analytical and numerical explorations has been aptly argued, the question remains to what extent do such pulses resemble pulses that can be generated by physical circuitry. 
This question was affirmatively answered already in [16] that evidenced the similarity between suitably constructed $\partial_{t} \mathrm{PE}$ and time-differentiated, ringing PE pulses, on the one hand, and the TD signatures generated by the circuits presented in [44] and [45], respectively, on the other hand.

The $\partial_{t}$ WP has an even higher similitude with physically generated pulses. In this respect, [21] has evidenced its likeness with the monocycle generated by the circuit introduced in [46], whereas [23] has shown its practical identity with the one generated by the solid-state implementation discussed in [24]. Based on the latter demonstrated congruence, $\partial_{t} \mathrm{WP}$ represents the monocycle of choice for modeling UWB antenna systems - any computer simulated prediction has very high chances of being amenable to a physical implementation since the feeding pulse generator is readily available.

\section{Conclusions}

The crucial role played in EM simulations by the excitation's causality was attested via theoretical arguments and numerical examples. Upon establishing the need for causal excitations, two families of pulses were constructed by starting from windowed-power (WP) and power-exponential (PE) unipolar prototypes. These prototypes were then used for deriving monocycles and pulses with almost rectangular spectra, and as envelops for constructing ringing pulses. The account constantly stressed the conceptual and practical benefits entailed by using these pulses and cogently demonstrated their superiority with respect to other excitations that are regularly used in antenna systems simulations. Two practical applications that particularly illustrate the opportunity of the time-windowed WP family were singled out. Firstly, the study promoted TD, time-windowed computational schemes as a means for eliminating the effect of the spurious reflections from the boundaries of computational domains and/or for drastically reducing runtimes. Secondly, upon noting the near-coincidence of this mathematical instrument with signatures that are generated by readily-available, solid-state pulse generators, the WP monocycle was put forward as an ideal excitation type in any design framework within the scope of UWB antenna systems.

\section{Acknowledgments}

The author expresses his gratitude to Dr. Martin Štumpf for pointing out the similarity between the WP and $\partial_{t} \mathrm{WP}$ signatures and (combinations of) TD convolutions of suitably chosen rectangular and triangular pulses, and for sharing with him the Matlab ${ }^{\circledR}$ codes demonstrating these features.

\section{References}

[1] MÜLLER-KIRSTEN, H. J. W. Electrodynamics. 2nd ed., New Jersey (USA): World Scientific Publishing Co. Pte. Ltd., 2011. ISBN: 9789814340731

[2] GARG, A. Classical Electromagnetism in a Nutshell, New Jersey (USA): Princeton University Press, 2012. ISBN: 9780691130187

[3] DE HOOP, A.T. A time-domain uniqueness theorem for electromagnetic wavefield modeling in dispersive, anisotropic media. The Radio Science Bulletin, 2003, vol. 2003, no. 305, p. 17-21. DOI: 10.23919/URSIRSB.2003.7909315

[4] DE HOOP, A. T. Handbook of Radiation and Scattering of Waves, London (UK): Academic Press, 1995. ISBN: 0122086554

[5] LAGER, I. E., DE HOOP, A. T., Causal pulses with rectangular spectral content: A tool for TD analysis of UWB antenna performance. IEEE Antennas and Wireless Propagation Letters, 2013, vol. 12, no. 1, p. 1488-1491. DOI: 10.1109/LAWP.2013.2289851

[6] PROAKIS, J. K., SALEHI, M. Digital Communications. 5th ed., Boston (USA): McGraw-Hill, 2008. ISBN: 9780072957167

[7] ADAMIUK, G., ZWICK, T., WIESBECK, W. UWB antennas for communication systems. Proceedings of the IEEE, 2012, vol. 100, no. 7, p. 2308-2321. DOI: 10.1109/JPROC.2012.2188369

[8] FEDERAL COMMUNICATIONS COMMISSION. First Report and Order April 2002. [Online] Cited 2021-01-05. Available at: https://transition.fcc.gov/Bureaus/Engineering_Technology/Orders /2002/fcc02048.pdf

[9] ZHANG, X., LARSON, L. E. ASBECK, P. M., Design of Linear RF Outphasing Power Amplifiers. Norwood (USA): Artech House, 2003. ISBN: 9781580533744

[10] CIATTAGLIA, M., MARROCCO, G. Investigation on antenna coupling in pulsed arrays. IEEE Transactions on Antennas and Propagation, 2006, vol. 54, no. 3, p. 835-843. DOI: $10.1109 /$ TAP.2006.869930

[11] DUMOULIN, A., AMMANN, M. J. Differentially-fed UWB slot antenna for direct board integration. In Proceedings of the 6th European Conference on Antennas and Propagation (EuCAP). Prague (Czech Republic), 2012, p. 765-768. DOI: 10.1109/EuCAP.2012.6205931

[12] DUMOUlin, A., JOHN, M., AMMANN, M. J., et al. Optimized monopole and dipole antennas for UWB asset tag location systems. IEEE Transactions on Antennas and Propagation, 2012, vol. 60, no. 6, p. 2896-2904. DOI: 10.1109/TAP.2012.2194686

[13] TRIVERIO, P., GRIVET-TALOCIA, S., NAKHLA, M. S., et al. Stability, causality, and passivity in electrical interconnect models. IEEE Transactions on Advanced Packaging, 2007, vol. 30, no. 4, p. 795808. DOI: 10.1109/TADVP.2007.901567

[14] WIDDER, D. V. The Laplace Transform. New Jersey (USA): Princeton University Press, 1941. ISBN: 9780691653693

[15] ŠTUMPF, M. Time-Domain Electromagnetic Reciprocity in Antenna Modeling. Hoboken (USA): Wiley, 2018. ISBN: 9781119466376

[16] LAGER, I.E., DE HOOP, A.T., KIKKAWA, T., Model pulses for performance prediction of digital microelectronic systems. IEEE Transactions on Components, Packaging, and Manufacturing Technology, 2012, vol. 2, no. 11, p. 1859-1870. DOI: $10.1109 /$ TCPMT.2012.2216266

[17] FRANCESCHETTI, G., TATOIAN, J., GIBBS, G. Timed arrays in a nutshell. IEEE Transactions on Antennas and Propagation, 2005, vol. 53, no. 12, p. 4073-4082. DOI: 10.1109/TAP.2005.859765 
[18] WIESBECK, W., ADAMIUK, G., STURM, C., Basic properties and design principles of UWB antennas. Proceedings of the IEEE, 2009, vol. 97, no. 2, p. 372-385. DOI: 10.1109/JPROC.2008.2008838

[19] DE HOOP, A. T., LAGER, I. E. Loop-to-loop pulsed electromagnetic field wireless signal transfer. In Proceedings of the 6th European Conference on Antennas and Propagation (EuCAP). Prague (Czech Republic), 2012, p. 786-790. DOI: 10.1109/EuCAP.2012.6205857

[20] ABRAMOWITZ, M., STEGUN, I. A. Handbook of Mathematical Functions, Mineola (USA): Dover Publications, 1968. ISBN: 9780486612720

[21] LAGER, I.E., VAN BERKEL S. L., Finite temporal support pulses for EM excitation. IEEE Antennas and Wireless Propagation Letters, 2017, vol. 16, p. 1659-1662. DOI: 10.1109/LAWP.2017.2662205

[22] WEISSTEIN, E. W. CRC Concise Encyclopedia of Mathematics, Boca Raton (USA): CRC Press LLC, 1999. ISBN: 9780849319457

[23] LAGER, I.E., ZITO, D. Pulsed EM field radio: The low-power, ultra-fast bridge to ubiquitous fiber networks. In Proceedings of the 13th European Conference on Antennas and Propagation (EuCAP). Krakow (Poland), 2019, p. 1-5. ISBN: 978-1-5386-8127-5

[24] PEPE, D., ALUIGI, L., ZITO, D. Sub-100 ps monocycle Pulses for $5 \mathrm{G}$ UWB communications. In Proceedings of the 10th European Conference on Antennas and Propagation (EuCAP). Davos (Switzerland), 2016, p. 1-4. DOI: 10.1109/EuCAP.2016.7481123

[25] LAGER, I. E., VOOGT, V., KOOIJ, B. J., Pulsed EM field, closerange signal transfer in layered configurations - a time-domain analysis. IEEE Transactions on Antennas and Propagation, 2014, vol. 62, no. 5, p. 2642-2651. DOI: 10.1109/TAP.2014.2307588

[26] ŠTUMPF, M. Receiving-antenna Kirchhoff-equivalent circuits and their scattering reciprocity properties. IET Microwaves, Antennas \& Propagation, 2016, vol. 10, no. 9, p. 983-990. DOI: 10.1049/iet-map.2016.0100

[27] ŠTUMPF, M. Coupling of impulsive EM plane-wave fields to narrow conductive strips: An analysis based on the concept of external impedance. IEEE Transactions on Electromagnetic Compatibility, 2018, vol. 60, no. 2, p. 548-551. DOI: 10.1109/TEMC.2017.2721445

[28] ŠTUMPF, M. Time-domain analysis of rectangular power-ground structures with relaxation. IEEE Transactions on Electromagnetic Compatibility, 2014, vol. 56, no. 5, p. 1095-1102. DOI: 10.1109/TEMC.2014.2305014

[29] DE HOOP, A. T., LAGER, I.E. Pulse shape distortion in closedwaveguide axial modal signal transfer - An analytic time-domain study. In Proceedings of the 8th European Conference on Antennas and Propagation (EuCAP). The Hague (Netherlands), 2014, p. 1531-1535. DOI: 10.1109/EuCAP.2014.6902074

[30] ŠTUMPF, M. Radar imaging of impenetrable and penetrable targets from finite-duration pulsed signatures. IEEE Transactions on Antennas and Propagation, 2014, vol. 62, no. 6, p. 3035-3041. DOI: $10.1109 /$ TAP.2014.2309964

[31] ŠTUMPF, M. The pulsed EM plane-wave response of a thin planar antenna. Journal of Electromagneticwaves and Applications, 2016, vol. 30, no. 9, p. 1133-1146. DOI: 10.1080/09205071.2016.1179132

[32] ŠTUMPF, M. The time-domain contour integral method - an approach to the analysis of double-plane circuits. IEEE Transactions on Electromagnetic Compatibility, 2014, vol. 56, no. 2, p. 367-376. DOI: 10.1109/TEMC.2013.2280297

[33] ŠTUMPF, M., ANTONINI, G., LAGER, I. E., Pulsed EM field transfer between a horizontal electric dipole and a transmission line A closed-form model based on the Cagniard-de Hoop technique. IEEE Transactions on Antennas and Propagation, 2020, vol. 68, no. 4, p. 2911-2918. DOI: 10.1109/TAP.2019.2935115
[34] ŠTUMPF, M., ANTONINI, G., LAGER, I.E., et al. Time-domain electromagnetic-field transmission between small-loop antennas on a half-space with conductive and dielectric properties. IEEE Transactions on Antennas and Propagation, 2020, vol. 68, no. 2, p. 938-946. DOI: $10.1109 /$ TAP.2019.2943323

[35] KNAB, J. Interpolation of band-limited functions using the approximate prolate series. IEEE Transactions on Information Theory, 1979, vol. 25, no. 6, p. 717-720. DOI: 10.1109/TIT.1979.1056115

[36] NATIONAL TELECOMMUNICATIONS AND INFORMATION ADMINISTRATION. Manual of regulations and procedures for federal radio frequency management. 2013 Edition, May 2013. [Online] Cited 2021-01-05. Available at: http://www.ntia.doc.gov/files/ntia/publications/redbook/2013/ May_2013_Edition_of_the_NTIA_Manual.pdf.

[37] LAGER, I.E., STASZEWSKI, R. B., SMOLDERS, A. B., et al. Ultra-high data-rate wireless transfer in a saturated spectrum - new paradigms. In Proceedings of the 44th European Microwave Conference (EuMC). Rome (Italy), 2014, p. 917-920. DOI: 10.1109/EuMC.2014.6986585

[38] MUR, G. Absorbing boundary conditions for the finite-difference approximation of the time-domain electromagnetic-field equations. IEEE Transactions on Electromagnetic Compatibility, 1981, vol. 23, no. 4, p. 377-382. DOI: 10.1109/TEMC.1981.303970

[39] MUR, G. Total-field absorbing boundary conditions for the timedomain electromagnetic field equations. IEEE Transactions on Electromagnetic Compatibility, 1998, vol. 40, no. 2, p. 100-102. DOI: $10.1109 / 15.673614$

[40] BERENGER, J.P. A perfectly matched layer for the absorption of electromagnetic waves. Journal of Computational Physics, 1994, vol. 114, p. 185-200. DOI: 10.1006/jcph.1996.0181

[41] BERENGER, J. P. Evanescent waves in PML's: Origin of the numerical reflection in wave-structure interaction problems. Transactions on Antennas and Propagation, 1999, vol. 47, no. 10, p. 1497-1503. DOI: $10.1109 / 8.805891$

[42] BERENGER, J. P. Numerical reflection from FDTD-PMLs: A comparison of the split PML with the unsplit and CFS PMLs. IEEE Transactions on Antennas and Propagation, 2002, vol. 50, no. 3, p. $258-265$. DOI: $10.1109 / 8.999615$

[43] DE HOOP, A. T., REMIS, R. F., VAN DEN BERG, P. M. The 3D wave equation and its Cartesian coordinate stretched perfectly matched embedding - a time-domain Green's function performance analysis. Journal of Computational Physics, 2006, vol. 221, p. 88-105. DOI: $10.1016 /$ j.jcp.2006.06.018

[44] ZITO, F., PEPE, D., ZITO, D. UWB CMOS monocycle pulse generator. Transactions on Circuits and Systems I: Regular Papers, 2010, vol. 57, no. 10, p. 2654-2664. DOI: 10.1109/TCSI.2010.2047751

[45] KIMOTO, K., KIKKAWA, T. Transmission characteristics of Gaussian monocycle pulses for inter-chip wireless interconnections using integrated antennas. Japanese Journal of Applied Physics, 2005, vol. 44, no. 4B, p. 2761-2765. DOI. 10.1143/JJAP.44.2761

[46] HAFIZ, M., KUBOTA, S., SASAKI, et al. A 2 Gb/s 1.8 pJ/bit differential BPSK UWB-IR transmitter using $65 \mathrm{~nm}$ CMOS technology. IEICE Transactions on Electronics, 2011, vol. E94-C, no. 2, p. 977-984. DOI. 10.1587/transele.E94.C.977

[47] BRACEWELL, R. N. The Fourier Transform and Its Applications, Boston (USA): McGraw-Hill, 2000. ISBN: 9780073039381 


\section{About the Authors...}

Ioan E. LAGER was born in 1962. He received his engineer degree (M.Sc.) from the University of Braşov, Romania, and PhD degrees from Delft University of Technology, the Netherlands (1996) and from the University of Braşov (1998). His research interests focus on applied electromagnetics, especially time-domain propagation and applications, and nonuniform array antennas. More recently, he also concentrated on new modalities for teaching classical electromagnetic field theory in under and post-graduate curricula.

\section{Appendix A: Proof of $\mathcal{F}[\mathbf{W P}(t)]$}

To begin with, it will be shown by induction that

$$
\begin{aligned}
& \mathcal{F}\left[\left[1-(x / a)^{2}\right]^{n} \Pi(x, a)\right]=2^{n} n ! a \sqrt{2 \pi} \frac{J_{n+1 / 2}(\omega a)}{(\omega a)^{n+1 / 2}} \\
& =\frac{2^{n+1} n ! a}{(\omega a)^{n}} \mathrm{j}_{\mathrm{n}}(\omega a) \text { for } n=1,2, \ldots
\end{aligned}
$$

in which $a>0$ is a real constant, $j_{n}$ is the spherical Bessel function of the first kind [20] and

$$
\begin{aligned}
& \Pi\left(x, x_{\mathrm{w}}\right) \stackrel{\text { def }}{=} H\left(x+x_{\mathrm{w}} / 2\right)-H\left(x-x_{\mathrm{w}} / 2\right) \\
& \text { for } x, x_{\mathrm{w}} \in \mathbb{R}, x_{\mathrm{w}}>0
\end{aligned}
$$

is the rectangle function. Note that in (A1) use was made of the definition of $j_{n}(\cdot)$ in [20].

The expression (A1) is directly verified for $n=1$ and $n=2$ by observing that $1-(x / a)^{2}$ represents the Welch function while $\left(1-(x / a)^{2}\right)^{2}$ the Connes function, their Fourier transforms being given in [22]. For demonstrating that the validity of (A1) implies its validity for $n+1$, the expression is transformed by applying the similarity theorem in the Fourier transform [47] that, since $a>0$, yields

$$
\mathcal{F}\left[\left[1-\xi^{2}\right]^{n} \Pi(\xi, 1)\right]=2^{n+1} n ! \frac{\mathrm{j}_{\mathrm{n}}(\omega)}{\omega^{n}}
$$

with $\xi=x / a$. By now making use of the identity

$\left(1-\xi^{2}\right)^{n+1} \Pi(\xi, 1)=-2(n+1) \int_{u=-\infty}^{\xi} u\left(1-u^{2}\right)^{n} \Pi(u, 1) \mathrm{d} u$ for $-1 \leqslant \xi \leqslant 1$

and using well-known properties of the Fourier transform, it is successively found that

$$
\begin{aligned}
& \mathcal{F}\left[\left(1-\xi^{2}\right)^{n+1} \Pi(\xi, 1)\right] \\
& =-2(n+1) \mathcal{F}\left[\int_{u=-\infty}^{\xi} u\left(1-u^{2}\right)^{n} \Pi(u, 1) \mathrm{d} u\right] \\
& =-2(n+1) \frac{1}{\mathrm{j} \omega} \mathcal{F}\left[\xi\left(1-\xi^{2}\right)^{n} \Pi(\xi, 1)\right] \\
& =-2(n+1) \frac{1}{\mathrm{j} \omega} \mathrm{j} \frac{\mathrm{d}}{\mathrm{d} \omega}\left\{\mathcal{F}\left[\left(1-\xi^{2}\right)^{n} \Pi(\xi, 1)\right]\right\} .
\end{aligned}
$$

Note that in deriving this equation use was made of the condition $\left.\mathcal{F}\left[\xi\left(1-\xi^{2}\right)^{n} \Pi(\xi, 1)\right]\right|_{\omega=0}=0$ which follows from $\xi\left(1-\xi^{2}\right)^{n}$ being a real, odd function (see [47]). Substituting (A3) in (A5) now yields

$$
\begin{aligned}
& \mathcal{F}\left[\left(1-\xi^{2}\right)^{n+1} \Pi(\xi, 1)\right] \\
& =2(n+1) \frac{1}{\omega} 2^{n+1} n ! \frac{\mathrm{d}}{\mathrm{d} \omega}\left[-\frac{\mathrm{j}_{\mathrm{n}}(\omega)}{\omega^{n}}\right] \\
& =2^{n+2}(n+1) ! \frac{1}{\omega}\left[\frac{n}{\omega^{n+1}} \mathrm{j}_{\mathrm{n}}(\omega)-\frac{1}{\omega^{n}} \frac{\mathrm{d}}{\mathrm{d} \omega} \mathrm{j}_{\mathrm{n}}(\omega)\right] \\
& =2^{n+2}(n+1) ! \frac{1}{\omega^{n+1}}\left[\frac{n}{\omega} j_{n}(\omega)-\frac{\mathrm{d}}{\mathrm{d} \omega} \mathrm{j}_{\mathrm{n}}(\omega)\right] .
\end{aligned}
$$

This, by using the formula (10.1.22) in [20], entails

$$
\mathcal{F}\left[\left(1-\xi^{2}\right)^{n+1} \Pi(\xi, 1)\right]=2^{n+2}(n+1) ! \frac{\mathrm{j}_{\mathrm{n}+1}(\omega)}{\omega^{n+1}}
$$

that, after scaling back for the original variable $x=a \xi$, concludes the proof by induction. It must be observed that (A1) is not defined at $\omega=0$. Once the validity of (A1) was established for $\mathbb{R} \backslash 0$, the Fourier transform is continued analytically via Abel's theorem [14] as

$$
\begin{aligned}
& \mathcal{F}(0)=\lim _{\omega \uparrow 0 ; \downarrow 0} \mathcal{F}(\mathrm{j} \omega)=\int_{-a}^{a}\left[1-(x / a)^{2}\right]^{n} \mathrm{~d} x \\
& =a \frac{2^{2 n+1}(n !)^{2}}{(2 n+1) !}
\end{aligned}
$$

the limits being equal because $\left[1-(x / a)^{2}\right]^{n}$ is a real, even function (see [47]). By now taking $a=t_{\mathrm{r}}$ and by employing a change of variables $x=t-t_{\mathrm{r}}$, (A1) implies that

$$
\widehat{\mathrm{WP}}(v, \mathrm{j} \omega)=\exp \left(-\mathrm{j} \omega t_{\mathrm{r}}\right) t_{\mathrm{r}} 2^{v} v ! \sqrt{2 \pi} \frac{J_{v+1 / 2}\left(\omega t_{\mathrm{r}}\right)}{\left(\omega t_{\mathrm{r}}\right)^{v+1 / 2}}
$$

that is used in the paper (see Eq. (4)).

The validity of (A8) is established by starting from

$$
\begin{aligned}
\int_{t=-\infty}^{\infty} \mathrm{WP}(v, t) \mathrm{d} t & =\int_{t=-\infty}^{\infty}\left(t / t_{\mathrm{r}}\right)^{v}\left(2-t / t_{\mathrm{r}}\right)^{v} H(t) H\left(2 t_{\mathrm{r}}-t\right) \mathrm{d} t \\
& =\left.t_{\mathrm{r}}^{-2 v}\left[t^{v} H(t)\right] \stackrel{(t)}{*}\left[t^{v} H(t)\right]\right|_{t=2 t_{\mathrm{r}}} \quad \text { (A10) }
\end{aligned}
$$

in which $\stackrel{(t)}{*}$ denotes time convolution. Taking the Laplace transform of the right-hand side term in (A10) yields

$$
\begin{aligned}
& \mathcal{L}\left[\left[t^{v} H(t)\right] \stackrel{(t)}{*}\left[t^{v} H(t)\right]\right]=\left(\frac{v !}{s^{v+1}}\right)^{2} \\
& =\frac{(v !)^{2}}{(2 v+1) !} \frac{(2 v+1) !}{s^{2 v+2}}
\end{aligned}
$$

that, in turn, implies the inverse Laplace transform

$$
\mathcal{L}^{-1}\left[\frac{(v !)^{2}}{(2 v+1) !} \frac{(2 v+1) !}{s^{2 v+2}}\right]=\frac{(v !)^{2}}{(2 v+1) !} t^{2 v+1} H(t) .
$$


By now using (A12) in (A10) it follows that

$$
\int_{t=-\infty}^{\infty} \mathrm{WP}(v, t) \mathrm{d} t=t_{\mathrm{r}} \frac{2^{2 v+1}(v !)^{2}}{(2 v+1) !} .
$$

An elementary change of variables and accounting for $\Pi(x, a)$ allows establishing that

$$
\int_{-a}^{a}\left[1-(x / a)^{2}\right]^{n} \mathrm{~d} x=\int_{t=-\infty}^{\infty} \operatorname{WP}(n, t) \mathrm{d} t
$$

with $t_{\mathrm{r}}=a$, which concludes the proof.
Note that (A13) also readily yields the expression of the total energy of a unipolar WP pulse (used in [21]). By observing that

$$
\int_{t=0}^{2 t_{\mathrm{r}}}[\mathrm{WP}(v, t)]^{2} \mathrm{~d} t=\int_{t=-\infty}^{\infty} \mathrm{WP}(2 v, t) \mathrm{d} t
$$

where use was made of (3) and of the finiteness of the pulse's support, (A13) entails that

$$
\int_{t=0}^{2 t_{\mathrm{r}}}[\mathrm{WP}(v, t)]^{2} \mathrm{~d} t=t_{\mathrm{r}} \frac{2^{4 v+1}(2 v !)^{2}}{(4 v+1) !} .
$$

